\title{
ON A MIXED BOUNDARY VALUE PROBLEM OF HARMONIC FUNCTIONS ${ }^{1}$
}

\author{
NORA PERNAVS
}

The mixed boundary value problem has been treated recently by several authors $[4 ; 5 ; 6 ; 7]$. In this note we give an existence proof using subharmonic functions.

Consider a 2-dimensional multiply connected domain $D$ with the boundary $\Gamma$, which consists of $k$ closed curves $\Gamma_{1}, \Gamma_{2}, \cdots, \Gamma_{k}$. The boundary curves $\Gamma_{i}(i=1,2, \cdots, k)$ are assumed to have continuous tangents.

A real-valued continuous function $f(\zeta)$ is defined on a part $\alpha$ of the boundary. (The boundary points are denoted by $\zeta$.) $\alpha$ consists of a finite number of disjoint closed curves or $\operatorname{arcs} \alpha_{i}$. If $\alpha_{i}$ is an arc, then we denote by $P_{i}^{\prime}, P_{i}^{\prime \prime}$ its two end points. $|f(\zeta)| \leqq M$.

The remaining part of $\Gamma$ we denote by $\beta$. Another real valued continuous function $g(\zeta)$ is defined on the closure of $\beta$.

The problem is to determine a function $u(z)$ in $D$, such that:

(a) $u(z)$ is harmonic in $D$,

(b) $\lim _{z \rightarrow \zeta} u(z)=f(\zeta)$ on $\alpha_{i}$ and $\lim \sup _{z \rightarrow \zeta}|u(z)| \leqq M$ at $\zeta=P_{i}^{\prime}, P_{i}^{\prime \prime}$,

(c) $\lim _{z \rightarrow \zeta} u(z)$ is continuous for $\zeta$ on $\beta$,

(d) $\partial u / \partial n=g(\zeta)$ for $\zeta$ on $\beta$, where the differentiation is along the inward normal $n$.

We shall treat first the problem by assuming $g(\zeta)=0 .{ }^{2}$ Since Neumann's problem can be solved for smooth boundary, the assumption $g(\zeta)=0$ on $\beta$ is not a restriction.

Define the class $\mathcal{F}$ of admissible functions $v(z)$ with the properties:

(a) $v(z)$ is continuous subharmonic in $D$,

(b) $\lim \sup _{z \rightarrow \zeta} v(z) \leqq f(\zeta)$ for $\zeta$ on $\alpha$,

(c) $\lim _{z \rightarrow \zeta} v(z)$ exists and is continuous for $\zeta$ on $\beta$,

(d) $\lim$ inf $\Delta v / \Delta n \geqq 0$ on $\beta$. The lim inf is taken along the normal pointing into the interior of $D$.

Received by the editors November 24, 1954 and, in revised form, April 20, 1955.

${ }^{1}$ This research was supported by the United States Air Force, through the Office of Scientific Research of the Air Research and Development Command. The project was supervised by Dr. Y. W. Chen.

The author is greatly indebted to the referee of this paper for his many valuable advices which lead to more precise formulations of some of the statements.

${ }^{2}$ The same problem was treated by $R$. Courant by the method of variational calculus $[2$, p. 40$]$. 
First, the class $\mathcal{F}$ is not empty, because all the constants $\leqq-M$ belong to it. Second, due to the property $d$, the functions in $\mathcal{F}$ have $M$ as an upper bound. To prove that, we make use of a theorem by E. Hopf [3], according to which any admissible subharmonic function $v$ of class $C^{\prime \prime}$ does not attain its maximum on $\beta$. If $v$ is only continuous but not necessarily differentiable, Hopf's theorem remains valid for $v$. For, Hopf's proof goes through also in this case if one replaces his condition $L(v) \geqq 0$ by that of continuous subharmonic functions $v$, and applies the maximum principle. Hence we have $v \leqq M$ in $D+\beta$, for all $v$ in $\mathcal{F}$.

The harmonic function $u(z)$ will be determined by a modification of the well known method of O. Perron [9] on the Dirichlet problem.

We define the function $u$ at each point $z$ in $D$ by

$$
u(z)=\text { l.u.b. } v(z) \text {, }
$$

where the l.u.b. is taken over all $v$ in $\mathcal{F}$. This definition is justified because the functions $v$ are bounded from above.

LEMMA 1. The function $u(z)$ is harmonic in $D$.

Proof. Suppose $v_{1}, v_{2} \in \mathcal{F}$. We shall show that the function $V(z)$ $=\max \left(v_{1}, v_{2}\right)$ also belongs to $\mathcal{F}$. It is easily seen that the function $V(z)$ has the properties $a$ and $b$. Consider a point $P(\zeta)$ on $\beta$. Since $v_{1}$ and $v_{2}$ are continuous at $P$, we have $\lim _{z \rightarrow 5} V(z)=\lim \max _{z \rightarrow 5}\left(v_{1}, v_{2}\right)$ $=\lim _{z \rightarrow 5}\left[\left(v_{1}+v_{2}\right)+\left|v_{1}-v_{2}\right|\right] / 2$, and hence $V$ is continuous at $P$. Let $Q(z)$ be a point on the inward normal at $P(\zeta)$, and let $\Delta v_{i}=v_{i}(Q)$ $-v_{i}(P), i=1,2$. It is easily seen that $\Delta V \geqq \min \left(\Delta v_{1}, \Delta v_{2}\right)$. From this follows lim inf $\Delta V / \Delta n \geqq 0$.

What is said for $V$ is also valid for $\max \left(v_{1}, v_{2}, \cdots, v_{n}\right)=V_{n}$. We may now carry out Perron's construction in the customary manner $[1$, p. 197$]$ by forming a maximizing sequence $\left\{V_{n}\right\}$ of subharmonic functions in an arbitrary disk $\Delta_{1}$, whose closure is contained in $D$. The functions $V_{n}^{\prime}$ which are equal to $V_{n}$ outside and on the boundary of $\Delta_{1}$, and equal to the Poisson integrals in $\Delta_{1}$, form a nondecreasing sequence which converges to a harmonic function in $\Delta_{1}$. This limit function is equal to $u(z)$. Since $\Delta_{1}$ is an arbitrary disk, the function $u$ is harmonic in $D$.

LEMMA 2. The function $u(z)$ determined by (1) satisfies $\lim _{z \rightarrow 5} u(z)$ $=f(\zeta)$ on $\alpha$, except possibly the end points.

Proof. To prove the lemma we have to show that lim $\sup _{z \rightarrow 5_{0}} u(z)$ $\leqq f\left(\zeta_{0}\right)+\epsilon$ and $\lim \inf _{z \rightarrow \zeta_{0}} u(z) \geqq f\left(\zeta_{0}\right)-\epsilon$ for all $\epsilon>0$ and $\zeta_{0}$ on $\alpha$. The 
first inequality can be proved in the same manner as it is known for the Dirichlet problem $[1$, p. 198].

To prove the second inequality consider a simply connected subdomain $\Delta$ contained in $D$, such that a part $\delta$ of its boundary is on $\alpha$. The remaining part of the boundary of $\Delta$ we denote by $\gamma$. We can solve the Dirichlet problem in $\Delta$ with the boundary function $F=f(\zeta)-\epsilon, \epsilon>0$, on $\delta$ and $F=v_{0}$ on $\gamma$, where $v_{0}$ are the values on $\gamma$ of an admissible function $v$. We obtain a harmonic function $H(z)$ in $\Delta$.

Consider the function $W=H$ in $\Delta$ and $W=v$ in the rest of $D . W$ is subharmonic in $D, \lim _{z \rightarrow \zeta} W \leqq f(\zeta)$ on $\alpha, W=v$ on $\beta$, therefore $W$ is in $\mathcal{F}$.

Now $W=f\left(\zeta_{0}\right)-\epsilon$ at $\zeta_{0}$. As an admissible function $W(z) \leqq u(z)$ and $\lim \inf _{z \rightarrow \zeta_{0}} u(z) \geqq W\left(\zeta_{0}\right)=f\left(\zeta_{0}\right)-\epsilon$. Hence $\lim _{z \rightarrow \zeta} u(z)=f(\zeta)$ for all points of $\alpha$, except $P_{i}^{\prime}, P_{i}^{\prime \prime}$.

LEMMA 3. $u(z)$ is continuous and $\partial u / \partial n=0$ on $\beta$.

Proof. We shall make use of conformal mapping. ${ }^{3}$ Consider a simply connected subdomain $\Delta^{\prime}$ contained in $D$. The boundary of $\Delta^{\prime}$ is a simple closed curve. It consists of two arcs, one denoted by $\delta^{\prime}$ is a closed subarc of $\beta$, while the other, denoted by $\gamma^{\prime}$, is an open arc which lies in $D$. We can map $\Delta^{\prime}$ conformally onto a semicircular domain $d$ so that $\boldsymbol{\gamma}^{\prime}$ goes into the circular arc and $\delta^{\prime}$ in to the bounding diameter. Denote this mapping by $S$.

Consider the maximizing sequence $\left\{V_{n}\right\}$, which was used for construction of $u$. Each $V_{n}$ has continuous boundary values $h_{n}$ on $\gamma^{\prime}$. By $S$ the function $h_{n}$ goes into a continuous function $\phi_{n}$ on the circular arc of $d$. With the boundary function $\phi_{n}$ and its symmetric extension on the other semicircle we obtain a harmonic function $H_{n}$ in the closed disk. $H_{n}$ is symmetric with respect to the diameter and has a vanishing normal derivative on the diameter.

Now we transform $H_{n}$ back to $\Delta^{\prime}$ by $S^{-1}$, and get a harmonic function $G_{n}$, which has a vanishing normal derivative on $\delta^{\prime} . G_{n}=V_{n}$ on $\gamma^{\prime}$. Since

$$
\lim \inf \left(\frac{\Delta V_{n}}{\Delta n}-\frac{\Delta G_{n}}{\Delta n}\right) \geqq \lim \inf \frac{\Delta V_{n}}{\Delta n}-\lim \sup \frac{\partial G_{n}}{\partial n} \geqq 0
$$

on $\delta^{\prime}$, we conclude that $G_{n} \geqq V_{n}$ in $\Delta^{\prime}$ (by making use again of Hopf's lemma [3]). The function equal to $G_{n}$ in $\Delta^{\prime}$ and to $V_{n}$ outside of $\Delta^{\prime}$ is an admissible function. Hence the functions $G_{n}$ form a maximizing sequence $\left\{G_{n}\right\}$, which converges to $u$. Hence $u$ is continuous and $\partial u / \partial n=0$ on $\delta^{\prime}$, consequently on $\beta$.

${ }^{3}$ This is the only step in the proof that can not be used to treat the case of more than 2 independent variables. 
Lemma 4. The function $|u(z)| \leqq M$ in $D+\Gamma$.

Proof. From Lemmas 2 and 3 by the boundary behavior of $u$ it follows that $u \leqq M$ in $D+\Gamma$. $u$ may not be defined at the end points $P_{i}^{\prime}, P_{i}^{\prime \prime}$. Since $u \geqq-M$ we have $|u| \leqq M$.

Uniqueness of the solution. Suppose that there exists a harmonic function $u_{1}(z)$ which solves the problem and is different from $u(z)$. Consider the function $U=u-u_{1} . U$ is harmonic in $D, \lim _{z \rightarrow 5} U=0$ on the curves and open $\operatorname{arcs} \alpha_{i}, U$ is continuous on $\beta$ and bounded at the points $P_{i}^{\prime}, P_{i}^{\prime \prime}$ and furthermore $\partial U / \partial n=0$ on $\beta$. We shall show that the maximum of $U$ is on $\alpha$. Suppose that the supremum of $U$ is $M_{1}$ and is equal to the supremum at a point $P$ which is one of the $P_{i}^{\prime}, P_{i}^{\prime \prime}$. Consider a small circle $r$ around $P$, such that no other $P_{i}^{\prime}, P_{i}^{\prime \prime}$ are in it. The part of $\beta$ inside the circle $r$ is denoted by $\beta_{r}$, the intersection of $D$ and $r$ by $d_{r}$. Let $m=\max U$ on $d_{r}, m<M_{1}$. By reflecting $d_{r}$ on $\beta_{r}$ (through a conformal mapping as in Lemma 3) we obtain a domain in which the harmonic function is bounded by the constant $m$ or zero, except at the point $P$. We can apply the extended maximum principle for harmonic functions $[8, p .115]$ to conclude that the supremum is not at $P$. The maximum is not on $\beta$ [3]. Therefore it is on $\alpha$ and $U \leqq 0$ in $D+\Gamma$. By considering the function $-U$ instead of $U$, we get $-U \leqq 0$. Both inequalities together imply $U=0$ or $u \equiv u_{1}$.

\section{BIBLIOGRAPHY}

1. L. Ahlfors, Complex analysis, New York, McGraw-Hill, 1953.

2. R. Courant, Dirichlet's principle, conformal mapping and minimal surfaces, New York, Interscience, 1950.

3. E. Hopf, A remark on linear elliptic differential equations of second order, Proc. Amer. Math. Soc. vol. 3 (1952).

4. J. H. Huth, Mixed boundary value problems in potential theory, Journal of the Franklin Institute vol. 257 (1954) pp. 121-124.

5. Y. Komatu, Eine gemischte Randwertaufgabe für einen Kreis, Proc. Japan Acad. vol. 28 (1952) pp. 339-341.

6. - Mixed boundary value problems, Journal of the Faculty of Science. Imperial University of Tokyo. Section I vol. 6 (1953) pp. 345-391.

7. Y. Komatu and Imsik Hong, On mixed boundary value problems, Kodai Math. Sem. Rep. (1953) pp. 65-76.

8. R. Nevanlinna, Uniformisierung, Berlin, Springer, 1953.

9. O. Perron, Eine neue Behandlung der ersten Randwertaufgabe für $\Delta u=0$, Math. Zeit. vol. 18 (1923) pp. 42-54.

WAYNE UNIVERSITY 